\title{
The Vibrio cholerae ToxR Regulon Encodes Host- Specific Chemotaxis Proteins that Function in Intestinal Colonization
}

\author{
Pradeep Selvaraj, Rohit Gupta, Kenneth M. Peterson* \\ Department of Microbiology and Immunology, Louisiana State University Health Sciences Center, Shreveport, United States
}

Received: October 06, 2015; Accepted: November 28, 2015; Published: December 03, 2015

*Corresponding author: Kenneth M. Peterson, Department of Microbiology and Immunology, Louisiana State University Health Sciences Center, Shreveport, United States, Tel: +1-318-675-5753; Fax: +1-318-675-5764; E-mail: kpeter@lsuhsc.edu

\begin{abstract}
Virulence gene regulation in Vibrio cholerae is under the control of the ToxR-ToxT regulatory cascade. Chemotaxis and net motility have been shown to influence the infectivity of Vibrio cholerae. $V$. cholerae toxR mutants do not synthesize proteins required for chemotaxis towards mucus. The inability of the toxR mutant strain to recognize and swim towards mucus is due to their failure to synthesize AcfB, a methyl-accepting chemotaxis protein. AcfB has previously been shown to be involved in intestinal colonization using the infant mouse model of cholera infection. Wild type $V$. cholerae recognizes galactose-6-sulfate in the capillary tube assay whereas $V$. cholerae acfB mutants fail to migrate into the capillary tubes. Vibrio strains carrying a mutation in tcpI, a ToxR regulated gene found within the Vibrio Pathogenicity Island (VPI), which encodes a methyl accepting chemotaxis protein are fully chemotactic towards mucus and galactose-6-sulfate.
\end{abstract}

Keywords: Vibrio cholerae; ToxR Regulon; Accessory colonization factor; Chemotaxis

\section{Introduction}

The Vibrio cholerae ToxR regulon encodes host specific chemotaxis proteins that function in intestinal colonization. Vibrio cholerae is the etiologic agent of the severe diarrheal disease cholera in humans. Following the ingestion of contaminated food or water, $V$. cholerae colonize the intestinal epithelium of the small intestine via a complex and poorly complex regulatory circuit that results in the spatial and temporal expression of the cholera toxin and toxin-coregulated-pilus genes. The end result of this process is efficient colonization of the small intestine and the acute watery diarrhea associated with Asiatic cholera [1-7]. To successfully colonize the human small intestine, the vibrios must penetrate the mucus gel and attach to and colonize the brush borders of the micro villi. A key component of this early phase of intestinal colonization is vibrio motility and chemotaxis [8-11]. Non motile vibrios are severely attenuated in the infant mouse model of intestinal colonization whereas, non-chemotactic $V$. cholerae mutants are not defective in intestinal colonization [811]. Remarkably, non-chemotactic $V$. cholerae dramatically outcompete wild type organisms. Non-chemotactic $V$. cholerae also demonstrate an altered colonization phenotype, i.e., wild type $V$. cholerae colonize only the lower small intestine whereas the non-chemotactic mutants colonize both the upper and lower small intestine [8]. This out-competition phenotype during in vivo infection requires the presence of Counterclockwise (CCW) biased flagellum [11]. Chemotaxis may also play an important role in the ability of $V$. cholerae to cause epidemics. Passage of $V$. cholerae through the gastrointestinal tract results in a transiently non-chemotactic state that increases their infectivity $[12,13]$. Understanding the precise role of $V$. cholerae chemotaxis in intestinal colonization is complicated by the presence of three chemotaxis operons and at least 43 methyl-accepting chemotaxis proteins [14]. Only one of the chemotaxis operons has been shown to be required for standard chemotaxis $[15,16]$. The function of the other two chemotaxis operons is not presently known.

We have previously shown that two Vibrio Pathogenicity Island (VPI) genes ( $a c f B, t c p I)$ under the control of the ToxR-ToxT regulatory cascade encode members of the methyl-accepting chemotaxis family of proteins $[17,18]$. V. cholerae strains with mutations in $a c f B$ were initially identified in a transposon library as being slightly defective in intestinal colonization [19]. TcpI was identified as a negative regulator of pilus synthesis [5,18]. In this report we find that $V$. cholerae strains bearing mutations in $a c f B$ were defective for chemotaxis towards mucus whereas $t c p I$ mutants displayed wild type levels of chemotaxis towards mucus.

\section{Materials and Methods}

\section{Media}

Luria broth (LB) was used for to culture both $V$. cholerae and E. coli strains. The LB was supplemented with antibiotics (ampicillin at $50 \mathrm{ug} \mathrm{mL}^{-1}$, streptomycin at $100 \mu \mathrm{g} \mathrm{mL}^{-1}$ ) or $0.1 \%$ arabinose as needed. Vibrio motility was confirmed using $0.3 \%$ LB soft agar [17].

\section{Bacterial strains}

Table 1 lists the bacterial strains employed in this study.

\section{Chemotaxis assay}

$V$. cholerae strains were cultured in $\mathrm{LB}$ broth $\mathrm{pH} 6.5$ at $30^{\circ} \mathrm{C}$ 


\begin{tabular}{|c|c|c|}
\hline Strains & Genotype & Source/reference \\
\hline \multicolumn{3}{|l|}{ Vibrio cholerae } \\
\hline 0395 & 01 classical, Str ${ }^{r}$ & (19) \\
\hline $0395 \Delta a c f B$ & $\Delta a c f B, \operatorname{Str}^{r}$ & This study \\
\hline $0395 \triangle a c f B / \mathrm{pACFB}$ & $\Delta a c f B / \mathrm{p} a c f B, \mathrm{Str}^{\mathrm{r}}, \mathrm{Amp}^{\mathrm{r}}$ & This study \\
\hline $0395 \Delta$ tox $R$ & $\Delta t o x R, \operatorname{Str}^{\mathrm{r}}$ & (19) \\
\hline $0395 \Delta t c p I$ & $\Delta t c p I, \mathrm{Str}^{\mathrm{r}}$ & This study \\
\hline $0395 \Delta$ cheW1 & $\Delta$ cheW1, Str ${ }^{r}$ & This study \\
\hline $0395 \Delta$ cheW2 & $\Delta c h e W 2, \operatorname{Str}^{r}$ & This study \\
\hline $0395 \Delta$ cheW3 & $\Delta$ cheW3, Str ${ }^{r}$ & This Study \\
\hline \multicolumn{3}{|l|}{ Escherichia coli } \\
\hline Top10 & 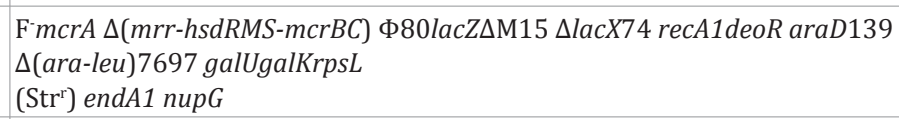 & Invitrogen \\
\hline DH5 $\alpha(\lambda$ pir $)$ & $\begin{array}{l}\text { supE4 DlacU169 (Ф80 lacZDM15) hsdR17 recA1 endA1 gyrA96thi-1 relA1 } \\
\text { גpir }\end{array}$ & Invitrogen \\
\hline $\operatorname{Sm} 10(\lambda$ pir $)$ & thi-1 thrleutonAlacYsupErecA::RP4-2Tc::Mu $\lambda$ pir R6K & $(37)$ \\
\hline Sy327( $\lambda$ pir) & $\Delta($ lac-pro $)$ arE $(\mathrm{Am})$ rifnalA recA56 $\lambda$ pir & $(37)$ \\
\hline \multicolumn{3}{|l|}{ Plasmids } \\
\hline pBAD & colE1 ori; araBAD promoter; $\mathrm{Amp}^{\mathrm{r}}$ & Invitrogen \\
\hline pACFB & acfB in $\mathrm{pBAD} ; \mathrm{Amp}^{\mathrm{r}}$ & This study \\
\hline pCVD442 & R6K ori;, mobRP4, bla, sacB & $(38)$ \\
\hline pGP704 & R6K ori; Amp ${ }^{\mathrm{r}}$ & (37) \\
\hline
\end{tabular}

containing the appropriate antibiotics overnight. The vibrios were then diluted 10 -fold in LB broth and cultured for 3 hours to maximize the number of motile cells. Vibrios were centrifuged at 5000X g and resuspended in Krebs-Ringer-Tris (KRT) buffer [8] to which $0.1 \%$ Triton X-100 was added to prevent excessive adherence of the bacteria to the glass surface. The vibrios were suspended in KRT at a concentration of $10^{7}$ bacteria/ $\mathrm{ml}$ and dispensed in $200 \mu \mathrm{l}$ aliquots into $1.5 \mathrm{ml}$ polypropylene tubes. A $25 \mu \mathrm{l}$ capillary tube (Drummond Scientific) heat sealed at one end and containing the taxin suspended in KRT was placed in the polypropylene tube approximately $0.5 \mathrm{~cm}$ below the surface of the Vibrio suspension. Following 60 minute incubation at $30^{\circ} \mathrm{C}$, the capillary tubes were removed and rinsed three times in KRT. The contents of the capillary tubes were then diluted in KRT and spread on agar plates to determine the number of colonies. All experiments were performed in triplicate on three separate occasions. Capillary tubes containing KRT were used as controls. The chemotactic response of $V$. cholerae to a specific taxin is expressed in terms of the relative response $\left(\mathrm{R}_{\text {che }}\right)$, i.e., the ratio of mean accumulation of vibrios in taxin containing capillaries to the mean accumulation of vibrios in control capillaries [8].

\section{Results}

\section{Chemotactic response of $V$. cholerae to mucus}

Chemotaxis represents an important mechanism whereby $V$. cholerae is able to colonize the small intestine. Thirty five years ago, Freter, discovered the importance of chemotaxis in the association of cholera vibrios with the intestinal mucosa using an elegant combination of in vitro/in vivo experiments [8-10]. More recently, the Camilli laboratory demonstrated that flagellarmediated chemotaxis contributes to $V$. cholerae colonization and infectivity [11]. Our finding that two ToxR/ToxT regulated genes ( $a c f B, t c p I$ ) found within the Tcp/Acf pathogenicity island encode members of the enteric methyl-accepting chemotaxis proteins $[17,18]$ prompted us to investigate their role in chemotaxis. Previous studies by Freter showed that motile bacteria guided by chemotactic gradients within mucus gel allowed the vibrios to penetrate efficiently into the deep layers of intervillous spaces [8]. To determine the roles of $\mathrm{AcfB}$ and $\mathrm{TcpI}$ in vibrio chemotaxis, $V$. cholerae strains containing in-frame mutations in acfB and tcpI were constructed. The $\Delta a c f B$ and $\Delta t c p I V$. cholerae strains were assayed for chemotaxis towards mucus using a capillary tube assay and the results are shown in Table 2.

\section{The role of the $V$. cholerae general chemotaxis machinery in response to mucus}

The completed genome of $V$. cholerae predicts a large number of proteins with amino acid similarity to known enteric related chemotaxis proteins [14]. The complexity of the $V$. cholerae chemotaxis system makes understanding its precise contribution to intestinal colonization an important undertaking. One characteristic of enteric MCPs that is shared by $A c f B$ is its ability to interact with the chemotaxis proteins of the general chemotaxis pathway. The interaction of enteric chemosensors with CheW 
Table 2: Chemotactic response of Vibrio cholerae to chemoattractants.

\begin{tabular}{|l|c|c|c|}
\hline Strain & Serine & Mucus & Galactose-6-sulfate \\
\hline 0395 & 18 & 12 & 12 \\
\hline $0395 \Delta t o x R$ & 18 & 3 & 4 \\
\hline 0395 acfB:: $\mathrm{Cm}^{\mathrm{R}}$ & 17 & 2 & 11 \\
\hline 0395 acfB:: $\mathrm{Cm}^{\mathrm{R}} / \mathrm{pACFB}$ & 17 & 11 & 11 \\
\hline $0395 \Delta t c p I$ & 17 & 12 & 3 \\
\hline $0395 \Delta$ cheW1 & 2 & 3 & 13 \\
\hline $0395 \Delta$ cheW2 & 18 & 11 & 12 \\
\hline $0395 \Delta$ cheW3 & 18 & 12 & 13 \\
\hline
\end{tabular}

Chemotactic response of $V$. cholerae to chemoattractants expressed in terms of relative response (Rche). This is the ratio of vibrio accumulation in chemoattractant-containing capillaries compared to capillaries containing buffer alone.

occurs via a Highly Conserved Domain (HCD) present in the cytoplasmic tail of these proteins [20-22]. The highly conserved domain of $A c f B$ is almost identical to other MCPs [17]. The possibility that AcfB interacts with $V$. cholerae CheW homologues seems likely since we know that $A c B$ influences vibrio swarm plate activity. Genome sequencing identified three $V$. cholerae genes encoding CheW homologues (cheW1, cheW2 and cheW3). It has also been shown that Chew1 is the dominant homologue (cheW1 null mutants fail to chemotax whereas cheW2 and cheW3 mutants are still chemotactic) $[15,16]$. To determine which of the three chemotaxis systems $A c f B$ signaling is coupled to, we generated $V$. cholerae strains containing mutations within cheW1, cheW2 and cheW3. Only the V. cholerae strain with a disruption in the cheW1 gene was found to be defective for vibrio chemotaxis to mucus. As can be seen in Table 2 strains with disrupted cheW1 fail to chemotax towards mucus.

\section{Chemotactic response to galactose-6-sulfate}

Mutations in $a c f C$ the gene directly downstream of $a c f B$ yields Vibrio strains with the same colonization defect as strains bearing $a c f B$ mutations [19]. Interestingly acfC encodes a protein related to sulfate binding proteins in E. coli. Since mucus is rich in sulfated sugars we tested the ability of $V$. cholerae to migrate towards a gradient of galactose-6-sulfate. Wild type $V$. cholerae is chemotactic towards galactose-6-sulfate whereas vibrios bearing an in-frame $a c f B$ deletion are defective for movement towards this taxin. As was noted above regarding $V$. cholerae chemotaxis towards mucus, only vibrios with a disruption of the cheW1 gene were defective for chemotaxis to galactose-6-sulfate (see Table 2).

\section{Discussion}

Motility and chemotaxis are utilized by pathogenic bacteria to colonize and invade the host [21]. Chemotaxis allows for Helicobacter pylori to invade the mucus lining of the stomach $[23,24]$ and Vibrio anguillarum uses chemotaxis to access the mucus of fish intestines [25,26]. V. cholerae which normally inhabits aquatic environments, possesses a remarkable repertoire of both inner membranes localized Methyl-Accepting Chemotaxis Proteins (MCPs) and soluble chemotaxis proteins $[14,15]$. Although $V$. cholerae is able to utilize chemotaxis to efficiently colonize the small intestines of humans the contribution of individual components of the vibrio chemotaxis system to this process is not well understood. This is especially true of the over 40 methyl-accepting chemotaxis proteins encoded by the $V$. cholerae genome [14]. The presence of $46 \mathrm{MCP}$-like proteins in $V$. cholerae suggests an impressive ability to respond to environmental signals. A key component of the $V$. cholerae virulence machinery lies within the TCP/ACF (VPI) pathogenicity island $[27,28]$. The toxin co-regulated pilus is absolutely required for intestinal colonization whereas the Acf proteins are needed for efficient colonization [19]. Two genes found within the VPI (acfB, tcpI) encode proteins related to methyl-accepting chemotaxis proteins $[17,18,29]$. MCPs form homodimers which span the bacterial inner membrane in order to transduce chemotactic signals to the cytoplasmic Che proteins such that the bacteria can respond with directed motility toward the toxins recognized by the individual MCP's [30,31]. Insertion mutations within acfB yield vibrio strains that are slightly deficient in colonization. Mutations within tcpI relieve pH mediated repression of TCP synthesis [19]. Strains containing tcpI mutations however are not defective for colonization in the infant mouse model of cholera infection [19]. We demonstrate that AcfB contributes to the ability of $V$. cholerae to recognize and swim toward a gradient of mucus whereas TcpI does not promote vibrio chemotaxis towards mucus.

We and others have used computer algorithms to predict that AcfB is structurally related to methyl accepting chemotaxis proteins $[17,29]$. Over expression of acfB in both $V$. cholerae and $E$. coli alters the swarm plate response of these strains. This suggests that AcfB is capable of interacting with the general chemotaxis machinery of both organisms. In this report we demonstrate for the first time that AcfB functions in $V$. cholerae chemotaxis. $V$. cholerae bearing mutations in $a c f B$ fail to respond to a gradient of mucus and also fail to recognize galactose-6-sulfate as a chemoattractant. Wild type V.cholerae respond with a positive chemotactic response to both of these substances. As with acfB, over expression of $t c p \mathrm{I}$ in $V$. cholerae alters the swarm plate response of these organisms in LB soft agar plates suggesting that $t c p I$ is able to interact with the general chemotaxis machinery. Unlike AcfB, however; TcpI does not appear to participate in the chemotactic response of $V$. cholerae to mucus/galactose-6-sulfate. TcpI is a $\mathrm{pH}$ dependent, 
negative regulator of TCP biogenesis. TcpI permits maximum synthesis of TCP in response to the $\mathrm{pH}$ of the culture medium. These findings and the relatedness of TcpI to MCPs, suggest that TcpI may "sense" pH. Capillary tube chemotaxis experiments in which V. cholerae 0395 and $V$. cholerae $0395 \Delta t c p I$ were exposed to KRT pH 6.5, KRT pH 7.4 and KRT pH 8.5 failed to demonstrate directed vibrio motility in response to $\mathrm{pH}$ (data not shown). Although regulation of TCP synthesis by TcpI appears to involve the ability of this inner membrane sensor protein to recognize $\mathrm{pH}$, the mechanism by which it affects this regulation and any possible role for Che proteins in this regulation remains obscure. Chemotaxis in bacteria is accomplished via a complex signal transduction system that permits sensory adaptation and relates the input signal to the flagellar motor [20-22, 30,31]. In many bacteria the signal transduction apparatus contains multiple sets of the proteins required for signal transduction [32]. The genome $V$. cholerae is predicted to encode 22 open reading frames that are homologous to che genes, most of the $V$. cholerae che genes are clustered in three regions on both chromosomes. The precise role of multiple sets of che genes in $V$. cholerae and other bacteria is not known [15]. Several che genes have been shown to affect cellular functions not related to chemotaxis. HlyB and TcpI were shown to be involved in hemolysin secretion and pilus biogenesis respectively $[17,33]$. Previous work examining the role of $V$. cholerae che paralogues in chemotaxis have shown that genes located in the che cluster II are responsible for $V$. cholerae chemotaxis $[15,16]$. The role of the genes encoded in clusters I and III have not been elucidated. In order to examine the role of the three che gene clusters in the $V$. cholerae chemotactic response to mucus we generated vibrio strains with mutations in cheW1, cheW2 and cheW3. Only mutations within cheW1 abolished vibrio chemotaxis towards mucus and galactose-6-sulfate.

The $V$. cholerae acfC gene is predicted to encode a periplasmic sulfate binding protein and is part of a polycistronic operon downstream of $a c f B$. Given its role in intestinal colonization, it seems likely that AcfC is involved in chemotaxis. Other periplasmic solute binding proteins such as the maltose binding protein and ribose binding protein have been shown to play a role in E. coli/Salmonella chemotaxis via interactions with methyl-accepting chemotaxis proteins [34]. Mucus is rich in sulfated molecules $[35,36]$ and thus we hypothesized that the ACF proteins may represent a sulfate "sensing" mechanism whereby $V$. cholerae could sense intestinal mucus and promote penetration of the mucus layer by directing chemotaxis toward sulfated sugars. Galactose is a common sulfated sugar found in mucus [36] and thus we tested galactose-6-sulfate for its ability to act as a chemoattractant. Parental V. cholera 0395 was capable of chemotaxis towards this sulfated sugar whereas $0395 \Delta a c f B$ and $0395 \Delta$ cheW1 were non-chemotactic towards galactose-6sulfate. These findings support out the hypothesis regarding the role of AcfB and AcfC in intestinal colonization such that AcfC binds galactose-6-sulfate followed by interaction with AcfB which in turn activates the chemotaxis signal transduction cascade mediated by Che proteins encoded by the che II gene cluster.

Recent efforts aimed at generating live-attenuated $V c$ vaccine strains suggest that that motility may play a role in the residual virulence of $V c$ strains lacking cholera toxin genes [39,40]. These studies emphasize the importance of understanding the role of vibrio motility and chemotaxis proteins in intestinal colonization, as this might have a practical impact on the development of efficacious vaccines for the prevention of cholera and may also give new direction to vaccine research for other enteric pathogens. The results presented here, shed light on a novel aspect of $V c$ pathogenesis and promote a clearer understanding of the contribution of the $V c$ chemotaxis signaling proteins in the intestinal colonization process.

\section{Acknowledgments}

This work was supported by Public Health Services grant AI49193 from the National Institutes of Health.

\section{References}

1. Kaper JB, Morris JG Jr, Levine MM. Cholera. Clin Microbiol Rev. 1995;8(1):48-86.

2. Sack DA, Sack RB, Nair GB, Siddique AK. Cholera. Lancet 2004;363(9404):223-33.

3. Barua B. Cholera. Edited by Dhiman Barua, William B. Greenough III Plenum Publishing Corporation, New York. 1992:1-36.

4. Faruque SM, Albert MJ, Mekalanos JJ. Epidemiology, genetics, and ecology of toxigenic Vibrio cholerae. Microbiol Mol Biol Rev. 1998;62(4):1301-14

5. Peterson KM. Expression of Vibrio cholerae virulence genes in response to environmental signals. Curr Issues Intest Microbiol. 2002;3(2):29-38.

6. Skorupski K, Taylor RK. Control of the ToxR virulence regulon in Vibrio cholerae by environmental stimuli. Mol Microbiol. 1997;25(6):1003-

7. Childers BM, Klose KE. Regulation of virulence in Vibrio cholerae: the ToxR regulon. Future Microbiol. 2007;2(3):335-44.

8. R Freter, B Allweiss, P C O’Brien, S A Halstead, M S Macsai. Role of chemotaxis in the association of motile bacteria with intestinal mucosa: in vitro studies. Infect Immun. 1981;34(1):241-9.

9. Freter R, O’Brien PC, Macsai MS. Role of chemotaxis in the association of motile bacteria with intestinal mucosa: in vivo studies. Infect Immun. 1981;34(1):234-40.

10. Freter R, O'Brien PC. Role of chemotaxis in the association of motile bacteria with intestinal mucosa: fitness and virulence of nonchemotactic Vibrio cholerae mutants in infant mice. Infect Immun. 1981;34(1):222-33

11. Butler SM, Camilli A. Both chemotaxis and net motility greatly influence the infectivity of Vibrio cholerae. Proc Natl Acad Sci U S A 2004;101(14):5018-23.

12. Merrell DS, Butler SM, Qadri F, Dolganov NA, Alam A, Cohen MB, et al. Host-induced epidemic spread of the cholera bacterium. Nature. 2002; $417(6889): 642-5$

13. Bina J, Zhu J, Dziejman M, Faruque S, Calderwood S, Mekalanos J. ToxR regulon of Vibrio cholerae and its expression in vibrios shed by cholera patients. Proc Natl Acad Sci U S A. 2003;100(5):2801-6.

14. Heidelberg JF1, Eisen JA, Nelson WC, Clayton RA, Gwinn ML, Dodson RJ, et al. DNA sequence of both chromosomes of the cholera pathogen Vibrio cholerae. Nature. 2000;406(6795):477-83. 
15. Boin MA, Austin MJ, Häse CC. Chemotaxis in Vibrio cholerae. FEMS Microbiol Lett. 2004;239(1):1-8.

16. Gosink KK, Kobayashi R, Kawagishi I, Häse CC. Analyses of the roles of the three cheA homologs in chemotaxis of Vibrio cholerae. J Bacteriol. 2002;184(6):1767-71.

17. Everiss KD, Hughes KJ, Kovach ME, Peterson KM. The Vibrio cholerae acfB colonization determinant encodes an inner membrane protein that is related to a family of signal-transducing proteins. Infect Immun. 1994;62(8):3289-98.

18. Harkey CW, Everiss KD, Peterson KM. The Vibrio cholerae toxincoregulated-pilus gene tcpI encodes a homolog of methyl-accepting chemotaxis proteins. Infect Immun. 1994;62(7):2669-78.

19. Peterson KM, Mekalanos JJ. Characterization of the Vibrio cholerae ToxR regulon: identification of novel genes involved in intestinal colonization. Infect Immun. 1988;56(11):2822-9.

20. Lux R, Shi W. Chemotaxis-guided movements in bacteria. Crit Rev Oral Biol Med. 2004;15(4):207-20.

21. Wadhams GH, Armitage JP. Making sense of it all: bacterial chemotaxis. Nat Rev Mol Cell Biol. 2004;5(12):1024-37.

22. Falke JJ, Hazelbauer GL. Transmembrane signaling in bacterial chemoreceptors. Trends Biochem Sci. 2001;26(4):257-65

23. Foynes S, Dorrell N, Ward SJ, Stabler RA, McColm AA, Rycroft AN, ET AL. Helicobacter pylori possesses two CheY response regulators and a histidine kinase sensor, CheA, which are essential for chemotaxis and colonization of the gastric mucosa. Infect Immun. 2000;68(4):2016 23.

24. Andermann TM, Chen YT, Ottemann KM. Two predicted chemoreceptors of Helicobacter pylori promote stomach infection. Infect Immun. 2002;70(10):5877-81

25. O’Toole R, Milton DL, Wolf-Watz H. Chemotactic motility is required for invasion of the host by the fish pathogen Vibrio anguillarum. Mol Microbiol. 1996;19(3):625-37.

26. O’Toole R, Lundberg S, Fredriksson SA, Jansson A, Nilsson B, WolfWatz $\mathrm{H}$. The chemotactic response of Vibrio anguillarum to fish intestinal mucus is mediated by a combination of multiple mucus components. J Bacteriol. 1999;181(14):4308-17.

27. Karaolis DK, Johnson JA, Bailey CC, Boedeker EC, Kaper JB, Reeves PR. A Vibrio cholerae pathogenicity island associated with epidemic and pandemic strains. Proc Natl Acad Sci USA. 1998;95(6):3134-9.
28. Kovach ME, Shaffer MD, Peterson KM. A putative integrase gene defines the distal end of a large cluster of ToxR-regulated colonization genes in Vibrio cholerae. Microbiology. 1996;142(Pt 8):2165-74.

29. Chaparro AP, Ali SK, Klose KE. The ToxT-dependent methyl-accepting chemoreceptors AcfB and TcpI contribute to Vibrio cholerae intestinal colonization. FEMS Microbiol Lett. 2010;302(2):99-105. doi: 10.1111/j.1574-6968.2009.01835.x

30. Mauriello EM. Cell biology of bacterial sensory modules. Front Biosci (Landmark Ed). 2013;18:928-43.

31. Sourjik V, Wingreen NS. Responding to chemical gradients: bacterial chemotaxis. Curr Opin Cell Biol. 2012;24(2):262-8. doi: 10.1016/j. ceb.2011.11.008.

32. Szurmant H, Ordal GW. Diversity in chemotaxis mechanisms among the bacteria and archaea. Microbiol Mol Biol Rev. 2004;68(2):301-19.

33. Alm RA, Manning PA. Characterization of the hlyB gene and its role in the production of the El Tor haemolysin of Vibrio cholerae 01. Mol Microbiol. 1990;4(3):413-25.

34. Kossmann M, Wolff C, Manson MD. Maltose chemoreceptor of Escherichia coli: interaction of maltose-binding protein and the tar signal transducer. J Bacteriol. 1988;170(10):4516-21.

35. Slomiany BL, Meyer K. Isolation and structural studies of sulfated glycoproteins of hog gastric mucosa. J Biol Chem. 1972;247(16):506270.

36. Slomiany B, Sominiany A. Mucus coat integrity and gastric disease In: The stomach: Physiology, Pathophysiology. Edited by W. Domschke, S.J. Konturek (Springer Verlag) Berlin. 1993:133-144.

37. Miller VL, Mekalanos JJ. A novel suicide vector and its use in construction of insertion mutations: osmoregulation of outer membrane proteins and virulence determinants in Vibrio cholerae requires toxR. J Bacteriol. 1988;170(6):2575-83.

38. Donnenberg MS, Kaper JB. Construction of an eae deletion mutant of enteropathogenic Escherichia coli by using a positive-selection suicide vector. Infect Immun. 1991;59(12):4310-7.

39. Kenner JR, Coster TS, Taylor DN, Trofa AF, Barrera-Oro M, Hyman T. Peru-15, an improved live attenuated oral vaccine candidate for Vibrio cholerae 01. J Infect Dis. 1995;172(4):1126-9.

40. Rui H, Ritchie JM, Bronson RT, Mekalanos JJ, Zhang Y, Waldor MK. Reactogenicity of live-attenuated Vibrio cholerae vaccines is dependent on flagellins. Proc Natl Acad Sci U S A. 2010;107(9):435964. doi: 10.1073/pnas.0915164107. 